\title{
Activités
}

\section{L'analyse collective des accidents du travail}

Une méthode d'analyse pour intégrer la dimension subjective et développer le genre professionnel

Group analysis of industrial accidents: a method incorporating the subjective aspect and examining different occupational constructs

\section{Claudia Osorio et Yves Clot}

\section{(2) OpenEdition}

Journals

Édition électronique

URL : http://journals.openedition.org/activites/2329

DOI : 10.4000/activites. 2329

ISSN : $1765-2723$

Éditeur

ARPACT - Association Recherches et Pratiques sur les ACTivités

Référence électronique

Claudia Osorio et Yves Clot, "L'analyse collective des accidents du travail ", Activités [En ligne],

7-1 | avril 2010, mis en ligne le 15 avril 2010, consulté le 19 avril 2019. URL : http://

journals.openedition.org/activites/2329 ; DOI : 10.4000/activites.2329

\section{(c) (i) (9)}

Activités est mis à disposition selon les termes de la licence Creative Commons Attribution - Pas d'Utilisation Commerciale - Pas de Modification 4.0 International. 


\title{
L'analyse collective des accidents du travail : une méthode d'analyse pour intégrer la dimension subjective et développer le genre professionnel ${ }^{1}$
}

\author{
Claudia Osorio \\ Université Fédérale Fluminense, département de psychologie. \\ Campus do Gragoatá, Bloco O , $2^{\circ}$ andar, Niterói, RJ, Brésil. \\ claudiaosorio@terra.com.br
}

Yves Clot

Conservatoire National des Arts et Métiers, Chaire de Psychologie du Travail et CRTD. 41 rue Gay-Lussac, 75005 Paris.

yves.clot@cnam.fr

\begin{abstract}
Group analysis of industrial accidents: a method incorporating the subjective aspect and examining different occupational constructs. The ACAT system (group analysis of industrial accidents), adopted as an intervention research method for hospital workers' health, has been developed since 2002 in a public hospital in the city of Rio de Janeiro. It aims at, on the one hand, analysing accidents by taking into account the realities of the work, and on the other hand, increasing the vitality of occupational constructs (Clot, 2008) in the hospital environment. The twin goals of this intervention research are to generate new knowledge about work in hospitals and to increase the involvement of doctors, nurses and other health professionals in managing their daily routines.

The method consists of asking a worker to recreate the scene of an accident by imagining himselves as an observer. First of all, he are asked to describe how the accident happened through an account of the movements and actions that led up to it. The researcher asks questions about the choices made about the various technical ways the actions might have been performed, what interruptions occurred and other details. The worker and researcher then record the sequence of events described in the form of a diagram, putting the emphasis on the worker's experience. Next, group discussions are held about several accidents, always with the objective of increasing workers' awareness of their own actions.

The methodology aims to contribute to a psychology of work that would be instrumental in encouraging individuals to reduce risk, through the development of occupational constructs. With this in mind, it uses a combination of the concepts of workers' health surveillance developed in Brazil, and French institutional and clinical work analysis.

The results obtained so far show that the method does indeed help workers to put themselves in the position of observers and co-analysts of their own work. It has also led to new understanding and new ways of performing tasks by breaking down entrenched habits that hinder the development of occupational constructs in the hospital environment.
\end{abstract}

KEYWORDS

Activity, industrial accidents, analysis of work.

1. Nous remercions Aurélie Fouilleul pour sa relecture de l'article. Nous remercions aussi les travailleurs de l'hôpital qui ont contribué à ce projet de diverses manières. 


\section{1.- Introduction}

Cet article met en débat une méthode d'analyse du travail et des accidents, produite dans une perspective transdisciplinaire, dont le but est de donner au travailleur la place de protagoniste de l'analyse.

Inscrit dans un processus de recherche-intervention ${ }^{2}$ sur la santé des travailleurs en milieu hospitalier, le dispositif d'Analyse Collective d'Accidents de Travail - l'ACAT - développé à partir de 2002 dans un hôpital public de la ville de Rio de Janeiro vise, d'une part, l'analyse des accidents en tenant compte du réel de l'activité de travail et, d'autre part, l'augmentation de la vitalité des genres d'activités en milieu hospitalier. La recherche est développée comme partie d'un contrat entre le secteur de l'hôpital responsable de la santé et la sécurité du personnel - la Commission de la Santé du Travailleur (CST) - et le département de psychologie de l'Université Fédérale Fluminense.

Dans cette recherche-intervention (Osario da Silva, 2002), l'objectif de production de connaissance sur le travail à l'hôpital s'ajoute à celui de l'augmentation de la participation des médecins, infirmiers et autres professionnels à la gestion de leur quotidien pour obtenir ainsi, dans la durée, une réduction de la fréquence et de la gravité des accidents provoqués par des instruments perforants coupants et l'exposition à des fluides biologiques. Les concepts et la méthodologie de l'analyse institutionnelle, de la surveillance en santé du travailleur et de la clinique de l'activité y sont utilisés.

L'analyse des accidents de travail déployée en association avec les travailleurs accidentés est ici exposée comme un instrument de formation dans le cadre du travail qui devra produire des effets transformateurs. Après deux ans de recherche, les résultats attendus étaient les suivants: une augmentation de «l'interférence » du travailleur avec son travail en raison du dialogue croissant que celui-ci a avec l'objet de son activité, ses collègues et la hiérarchie du service auquel il appartient, surtout pour ceux qui participent à l'analyse de leur accident, un accroissement de la participation des travailleurs aux actions qui visent la production de changements des «façons de faire » et des améliorations des conditions de travail concernant la santé du travailleur dans le domaine de la santé.

Au cours de cette recherche, d'août 2003 à avril 2005, 79 accidents ont été analysés et deux ateliers de discussion de ces accidents et de la procédure d'analyse adoptée, l'ACAT, ont été réalisés.

Des changements caractérisant le développement ou l'accroissement de la relation du travailleur avec son travail ont été observés dans la description du processus de travail entre la première mise en scène et la discussion de groupe.

La méthode d'analyse collective des accidents est produite dans un dialogue entre les concepts et l'expérience développée sur le terrain mentionné, mais les possibilités d'utilisation de la méthode ne se restreignent ni à l'analyse et à la prévention d'accidents par objets perforants coupants, ni à l'hôpital. Nous pensons, bien au contraire, que cette méthode est appropriée pour obtenir un accroissement de la participation et du pouvoir d'agir de groupes de travailleurs de diverses professions et spécialités et pour l'analyse de plusieurs types d'accidents et incidents. Les travailleurs ne savent pas de quoi ils sont capables sans l'expérimentation. Mais l'expérimentation exige prudence, et ces dispositifs d'analyse du travail, que nous développons, doivent offrir aux travailleurs des possibilités de vivre des expériences les incitant à en oser d'autres.

\section{2.- Les objectifs de la recherche}

Dans l'hôpital désormais considéré, il existe un service responsable de la santé et de la sécurité des employés, la CST ci-dessus mentionnée, créée par une direction sensible aux débats menés par le syndicat des médecins et le Conseil Professionnel de Médecine. Cette structure n'est pas prévue dans l'organigramme officiel du Ministère de la Santé et n'existe pas habituellement dans les organismes

2. Cette recherche a reçu l'aide financière de la Faperj. L'équipe de recherche était également composée d'étudiants de l'UFF (Université Fédérale Fluminense) dont trois ont bénéficié de bourses d'initiation scientifique financées par l’Université et la Faperj. 
de l'administration publique.

L'hôpital public au Brésil est confronté à des difficultés d'ordre divers: il ne jouit pas de tradition de participation, mais bien au contraire, d'une centralisation des décisions. En effet, la représentation syndicale est fragile et elle n'est ni substituée ni alliée à une structure additionnelle de quelque autre forme d'organisation politique. Il n'existe pas de tradition de recours à l'ergonomie dans la gestion du travail. Nous observons depuis assez longtemps, dans les hôpitaux, que le personnel a nettement cessé de croire à la possibilité que les collectifs de travail puissent avoir une influence dans l'organisation de leurs propres activités et nous observons par conséquent une prédominance d'un cadre défensif face à l'organisation du travail.

À la recherche d'un recours pour aller au-delà des difficultés imposées par cette réalité, la CST a choisi d'offrir des projets formateurs qui s'appuient sur un problème à résoudre, clair et bien défini, avec des perspectives immédiates en termes de production de solutions pratiques. Dans cette perspective, nous avons considéré que l'analyse et la prévention des accidents de travail par l'exposition aux perforants-coupants et aux fluides biologiques pourraient configurer une stratégie adéquate pour la création et l'installation de dispositifs de formation. De tels dispositifs pourront produire une réduction de la fréquence et de la gravité des accidents, mais aussi un développement des relations entre les professionnels de l'hôpital et leur travail.

En tant que proposition pédagogique de transfert de connaissance et d'informations, le programme de contrôle et de prévention d'accidents de cet hôpital est né en 1996 lors d'un séminaire sur la santé du travailleur. Par la suite furent amorcés: des actions de formation du personnel hospitalier relatives à la biosécurité, l'accueil des travailleurs accidentés, pour l'évaluation médicale des risques de contamination et l'administration de la prophylaxie adéquate; ainsi que la notification des accidents de travail. À partir de 1997, les données relatives aux accidents notifiés purent être enregistrées.

En 1998, ont débuté les analyses qualitatives des accidents notifiés à travers des témoignages venant des travailleurs accidentés (Chaves, S. M. C., Osório-da-Silva, C., Salvatierra, M. A., Muller, A. M. C., Ferrari, W., Nunes, J. et al., 1999; Osorio da Silva \& Oliveira, 1999).

Premièrement, bien que les travailleurs, dans leur grande majorité, aient pris connaissance des normes de sécurité actuellement recommandées pour le travail en hôpital (CDC, 1989), cette connaissance n'a pas été suffisante pour garantir le suivi de ces normes. Deuxièmement, de nombreux accidents survenaient au cours d'activités, d'actions dans lesquelles les travailleurs avec des fonctions très différentes se trouvaient étroitement liés, rendant ainsi impossible une analyse limitée au poste de travail où s'était passé l'accident. Troisièmement, les travailleurs, et en particulier le personnel infirmier, citaient comme étant un facteur important pour expliquer un comportement risqué, "l'habitude bien apprise » due à leur période de formation comme infirmier (ou aide-soignante). Et, en dernier lieu, les travailleurs affirmaient que leur engagement vis-à-vis du malade prenait le pas sur la préoccupation concernant leur propre sécurité ${ }^{3}$. La notion d'habitude représentait une explication fréquente pour justifier l'acte de re-capuchonner une aiguille déjà utilisée. Approximativement $20 \%$ des accidents enregistrés au cours des années 1997-1998 étaient de ce type (Chaves et al, 1999). À la suite de l'allégation de l'habitude bien ancrée dans sa pratique, le professionnel ajoutait: «j'ai peur de blesser quelqu'un, circulant dans l'infirmerie avec une aiguille décapuchonnée en main » ou bien encore: «j'étais occupé à soigner rapidement un patient et je n'ai pas suffisamment fait attention à ce que je faisais, et voilà, j'ai fait de la façon dont j'ai appris il y a bien longtemps et j'ai remis le capuchon sur l'aiguille.»

Les précautions universelles qui composent la stratégie de biosécurité en vigueur dans les hôpitaux ont été développées par le CDC en 1985 (CDC, 1989) et ont dès lors été intégrées dans la formation

3. Au moment de la recherche, la majorité du personnel infirmier de cet hôpital y travaille depuis 20 ans ou plus. Pendant leur formation académique, il n'y avait ni SIDA, ni les règles actuelles de biosécurité, ni les boîtes renforcées pour déposer les aiguilles. La consigne était de remettre le capuchon sur les aiguilles usagées pour éviter des accidents avec les résidus hospitaliers. Il faut aussi noter que le personnel infirmier est composé de infirmiers (ères) diplômés, de techniciens et d'aides soignant(e)s. 
de base du personnel de santé. Les entraînements développés à l'hôpital où nous faisions notre recherche ont été implantés par la CST en 1996.

Cependant, on constate, outre la non-observation de ces normes, qu'il y a une récurrence d'actions antérieurement préconisées - comme celle de remettre le capuchon des aiguilles - , caractérisant peut-être une cristallisation de gestes qui rend difficile l'élaboration de nouvelles normalisations (Canguilhem, 1999), d'une re-création qui serait un signe de la vitalité du genre professionnel.

Cette expérience et les résultats d'autres recherches (Carvalho \& Leite, 1999; Chaves, et al, 1999; Grosjean \& Lacoste, 1999; Muniz, 2000; Osorio da Silva, 1994; Pitta, 1989; Rego, 1993; Vega, 2000) nous permettent d'affirmer que l'activité de travail doit être analysée en tenant compte nonseulement de ce qui est fait mais aussi des conflits vécus par le travailleur entre les exigences alliées à la réalisation de son travail et les ressources subjectives qu'il déploie pour obtenir une solution. Dans cette étude, un conflit a été constaté entre les prescriptions techniques se rapportant au respect des normes de biosécurité et un sens attribué au travail, en l'occurrence celui de s'occuper des autres avant soi. Nous avons aussi observé, qu'entre le travail prescrit - techniquement correct - et le travail réalisé, s'interpose ce que le professionnel appelle l'habitude: le travailleur agit selon un apprentissage antérieur, automatisé, automatisation qui le débarrasse des plusieurs décisions. Il faut toujours faire des efforts pour trouver du sens à ces gestes difficiles à comprendre et s'interroger sur ces hypothèses de cristallisation de l'habitude. Ou, mieux, il faut trouver des chemins pour défaire ces cristallisations et développer l'expérience, pour qu'elle devienne le moyen pour vivre d'autres expériences.

À partir de ce constat, un objectif a été défini : élaborer des méthodes d'analyse des accidents du travail en conformité avec le milieu hospitalier qui permettraient le développement créatif de l'expérience. En reconnaissant les conflits qui caractérisent les soins aux malades et en renouvelant les moyens subjectifs dont le travailleur dispose pour recréer quotidiennement sa pratique, cette méthode devrait intervenir positivement dans la re-création de contenus cognitifs. Ainsi, on re-créerait également la pratique traditionnelle d'analyste du travail: le spécialiste quitte le rôle de celui qui conseille pour un autre, celui qui partage les voies et les solutions.

Nous considérons l'augmentation du pouvoir d'agir du travailleur comme le principal objectif de la méthode. Cet accroissement du pouvoir d'agir exige le développement de nouveaux engagements subjectifs, de sujets susceptibles de se mesurer à de nouvelles et à d'anciennes situations, à leur propre expérience et aussi à celle des autres. Dans cette perspective, le principal observateur de l'activité de travail doit être le travailleur lui-même et non pas le spécialiste en analyse du travail. Le spécialiste doit donc devenir l'instrument d'analyse (Clot, 1999, pp. 73-75) favorable au déplacement du travailleur sur une posture d'observation de sa propre activité.

\section{3.- La « boîte à outils » conceptuelle}

La méthodologie proposée s'inspire, en partie, du travail d'Ivar Oddone (Oddone, Re, \& Briante, 1981) en cherchant à contribuer à une psychologie du travail qui soit l'instrument d'une mobilisation subjective tournée vers la suppression du risque, par le développement des genres professionnels (Clot, 2006). Dans ce but, elle articule des concepts de l'analyse institutionnelle française, de la surveillance en santé du travailleur développée au Brésil et de la clinique de l'activité.

L'analyse institutionnelle française nous propose la méthodologie de la recherche-intervention où le concept de la multiréférentialité est central et «n'est pas une simple collection de disciplines juxtaposées. Elle se réfère à diverses méthodes et à l'utilisation de certains concepts déjà existants afin de construire un nouveau domaine de cohérence » (Lourau, 1993, p. 10). En emboîtant le pas à la formulation de l'analyse institutionnelle (Lourau, 1993), on considère que tout processus de recherche participe à la production d'une réalité qui n'est jamais statique et achevée. Cette participation du chercheur à la production de la réalité qu'il étudie doit être constamment analysée. En utilisant les 
concepts de la clinique de l'activité, on peut dire que la recherche est elle-même une contribution à la stylisation du genre professionnel du métier analysé.

Dans l'histoire de la clinique de l'activité (Clot, 2001, p. 8), nous faisons références à I. Oddone; ce dernier, dans ses travaux auprès des ouvriers de Fiat a eu le mérite et l'originalité d'évaluer l'impasse que la simple dénonciation des conditions de travail inacceptables produisait. Il s'est tourné vers la recherche des moyens dont disposaient les travailleurs eux-mêmes, pour agir sur leur travail; moyens insoupçonnables jusqu'alors, qu'ils auraient pu utiliser pour promouvoir et protéger leur propre santé. I. Oddone cherchait des moyens de seconder les collectifs de travail dans sa tentative de maintenir et d'amplifier son rayon d'action sur le travail: il cherchait une psychologie du travail autre que celle du spécialiste qui «traite » ou « conseille» (Oddone et al., 1981). Nous reprenons cet objectif: construire des formes pour seconder les travailleurs de la santé dans leur projet de transformation des conditions de travail à l'hôpital. Cet objectif devient important dans la mesure où nous faisons l'hypothèse que cette transformation entraînera des résultats positifs sur les malades dont ils s'occupent.

En reconnaissant le besoin déjà indiqué par I. Oddone (Oddone et al., 1981), de développer des méthodologies qui permettent de regarder l'activité de travail comme un processus en devenir constant, la clinique de l'activité propose deux méthodes: la reformulation des instructions au sosie - présentée auparavant par I. Oddone (Clot, 1995) - et l'autoconfrontation, développée comme l'autoconfrontation croisée (Clot, 1999). Ces méthodes sont fondées sur certains postulats: la connaissance rendue possible au cours des transformations toujours en tant que connaissance provisoire; la recherche en tant qu'intervention, la relation transversale entre sujet singulier et sujet collectif, sans dichotomie interne (individuelle, subjective) et externe (sociale); le conflit et la controverse en tant que moteur de production de singularités - ou, dans le langage de la clinique de l'activité, de la stylisation des genres - et en tant que constitutifs de l'activité de travail. La clinique de l'activité définit, comme objectif de ces méthodes, la production de nouvelles subjectivités sans éloigner la pensée de l'action; penser différemment c'est déjà agir de façon innovatrice.

Le désir de transformer les relations entre le processus de travail et les processus de production de subjectivités à l'hôpital est motivé par la reconnaissance d'une obligation éthique. Tous ceux qui sont en mesure d'intervenir dans les collectifs, favorisant la production de nouveaux sujets autonomes, réglés par la logique de la santé et de la vie ne peuvent pas éviter de participer à cet effort. ${ }^{4} \mathrm{Par}$ subjectivités, on entend les modes de penser, agir et sentir qui sont aussi bien personnels que collectifs; ils sont historiquement produits et nous traversent dans un entrecroisement toujours singulier. Partant de cette conception, il n'y a pas de dichotomie entre individuel et collectif: les valeurs collectives traversent chacun de nous dans des configurations singulières et jamais figées en formes achevées. La subjectivité se produit dans l'activité en même temps qu'elle produit l'activité.

Dans le domaine de la santé publique, et spécifiquement de la surveillance en santé du travailleur (Machado, 1997 ; Minayo-Gomez, \& Thedim-Costa, 1997), l'action interdisciplinaire, avec la participation active des travailleurs pour la transformation des processus de travail, est volontiers préconisée.

Nous soulignons comme objectifs concomitants de la méthode proposée: l'attention à chaque travailleur accidenté en considérant sa situation toujours singulière, de manière collective et individuelle; la formation conjointe de chercheurs et analystes du travail et de travailleurs de la santé; la production d'information pour l'action; l'amélioration des conditions de santé et de sécurité au travail dans l'hôpital; et la gestion démocratique du service de santé du travailleur. L'hybridation de logiques et de domaines d'actions traditionnellement séparés est ici recherchée.

Notre « boîte à outils » est un référentiel qui allie des concepts ou des outils théoriques appartenant,

4. Pour cette affirmation, on pense à Félix Guattari et ses propositions pour une nouvelle Écosophie (1990). Parmis les brésiliens, Gastão W. de S. Campos (1994 et 2000), en parlant spécifiquement du système de santé publique au Brésil et en profitant de la référence théorique de l'analyse institutionnelle, indique le besoin de dépasser les dénonciations et d'élaborer de nouvelles formes de gestion de l'assistance, en produisant de nouvelles subjectivités. 
à l'origine, à divers domaines. À partir de multiples références, nous construisons donc un nouveau domaine de cohérences. Le choix de ces outils théoriques est surtout un choix en rapport avec une conception de l'homme en tant qu'être capable de laisser une marque de sa participation et d'intervenir dans sa propre histoire - et une conception du travail, processus collectif et singulier de création et recréation de l'histoire d'un métier, de l'activité de travail en tant que processus de production, non seulement de choses ou de services, mais aussi de subjectivités.

Cependant, les demandes présentées par l'hôpital à la CST, lieu institué et instituant où commencent les interventions ici décrites, sont motivées par le vécu de souffrance et arrivent sous forme de plaintes. Les travailleurs ou les équipes des services cherchent un soulagement à leur douleur. Ainsi donc, l'accident de travail a été choisi comme départ d'une méthodologie d'analyse qui porte autant sur l'accident que sur l'activité de travail.

\section{4.- L'analyse collective des accidents de travail - L'ACAT}

Dans la méthode d'analyse collective d'accidents, la tâche consiste à élucider, pour l'autre et pour soi-même, les questions sur le parcours des événements qui aboutissent à un accident. Cette élucidation est le fruit de déplacements qui se déroulent dans une relation dialogique. Dans celle-ci, le travailleur accidenté, en tant que porteur d'expérience, s'adresse à lui-même, à ses collègues et à l'analyste. L'analyste, à son tour, en tant que sujet de connaissances formalisées, dans son analyse, s'adresse à lui-même, à ses pairs et aux travailleurs impliqués.

Dans la construction de l'analyse de l'accident, nous remontons le temps pour repérer les multiples situations qui se sont enchaînées et qui ont débouché sur l'événement considéré. En déplaçant le travailleur et son groupe vers la position d'observateurs de leur propre travail, l'expérience constituée jusque-là devra servir d'instrument pour le renouvellement du mode opératoire objectif et subjectif ; le genre professionnel (Clot, 1999) en question peut être renouvelé, avec pour résultat l'augmentation de la capacité d'agir des travailleurs en ce qui concerne notamment la prévention d'accidents.

L'analyse est proposée à tous les professionnels qui se sont accidentés et qui ont porté ce fait à la connaissance du service d'infectiologie de l'hôpital ou de la CST. Ces professionnels peuvent être, le plus souvent des médecins du staff, des médecins en formation et des étudiants en médecine; des infirmiers (ères), des techniciens, et des aides soignant(e)s; le personnel des laboratoires d'analyses cliniques; et des personnels de nettoyage. Ces professionnels ont été contactés par téléphone pour fixer un rendez-vous avec un membre de la CST, en l'occurrence ici un étudiant de psychologie impliqué dans la recherche. L'analyse a lieu au poste de travail de l'accidenté, ce qui doit favoriser la description de la mise en scène. À l'arrivée sur le lieu de travail de l'accidenté, l'analyste présente l'objectif de l'entretien, et met en lumière l'importance de l'aide de chacun pour la réduction des accidents.

Tout d'abord, l'analyste demande à l'accidenté son aide au nom de la CST qui a en effet la responsabilité de faire l'analyse des accidents, dans le but de proposer des actions de prévention. La consigne est alors: Est-ce que vous pourriez m'aider? On pourrait construire ensemble l'analyse de votre accident et faire des suggestions pour améliorer les conditions de travail à l'hôpital?

Dès qu'il accepte d'y participer, la première étape de l'ACAT est une mise en scène de l'accident qui permet de reconstruire la mémoire de l'accident aussi bien que la mémoire des règles et des moyens qui orientent le processus dans lequel a eu lieu l'accident. Dans ce but, l'analyste demande au professionnel accidenté de lui montrer comment l'accident est arrivé. Il doit montrer comment l'accident a eu lieu en mettant en scène les mouvements et actions qui ont abouti à l'accident. On observe, dans la plupart des cas, que le professionnel commence à décrire oralement la succession des faits. L'analyste doit alors lui demander de prendre les outils pour montrer comment il a réalisé l'intervention technique, d'aller sur le lieu où l'accident s'est produit, de faire comprendre comment il a occupé l'espace, et de détailler chaque mouvement. L'analyste du travail lui pose des questions 
sur les choix effectués quant aux divers chemins techniques possibles, les interruptions subies et les autres événements d'abord naturalisés. On s'intéresse également aux règles techniques, éthiques et de sécurité car il peut y avoir conflit entre elles. Le souvenir de l'accident et des normes n'est pas seulement récupéré, mais construit au cours du dialogue entre l'analyste et l'accidenté. L'analyste est un élément permettant l'éloignement nécessaire à la réflexion et à la construction de nouvelles formes de relations avec le travail.

Il y a donc une confrontation de diverses expériences et connaissances. Le fait que l'analyste n'appartienne pas au même métier permet un nouveau regard et force un besoin d'explication qui, entre pairs, n'aurait pas été nécessaire.

Dans cette approche, il convient de privilégier le «comment» dans le dialogue. Il faut analyser les conditions de l'occurrence de l'accident par rapport à la relation du professionnel envers le malade, ses collègues, la hiérarchie et les conditions matérielles dans lesquelles s'était réalisée la tâche. Il appartient à l'analyste d'éviter le « pourquoi ». Cette orientation a pour objectif d'éviter que les participants ne se perdent en justifications en ce qui concerne les choix qu'ils sont amenés à faire. Le « comment » induit à recréer dans la mémoire les attributs qui composent les genres professionnels, alors que le «pourquoi » rend propice l'évaluation des voies correctes ou erronées, préconisées ou non, ce qui conduit à rechercher des causes et des coupables pour l'accident. Il peut être aussi intéressant de travailler avec le " pour que », qui peut devenir un élément déclencheur du surgissement des aspects liés aux différents objectifs qui traversent une tâche.

La deuxième étape consiste à construire un dessin schématique des diverses lignes et croisements qui mènent à l'accident. Ce diagramme doit être construit collectivement et son exécution doit être attribuée à l'analyste, en privilégiant l'expérience du travailleur.

Le rôle de l'analyste dans ce travail est délicat. Il se doit d'être très attentif au cours de ses interventions pour ne pas introduire dans l'analyse des mots et des images qui soient les siens, et qui sont impropres à l'activité en analyse, ses genres et styles.

La situation engendrée, celle de décrire l'action pour l'analyste et pour son ou ses collègues, de crayonner et de visualiser à travers un dessin sur papier le croisement des événements qui construit une activité qui éventuellement pourrait paraître simple, produit un effet de distance vis-à-vis de soimême. Cette dynamique en soi, permet en même temps de connaître et de recréer son mode singulier d'agir.

Avec le diagramme esquissé, chaque participant recevra le matériel nécessaire pour le copier et l'emporter chez soi dans le but de l'élaborer à nouveau, constituant ainsi la troisième étape.

Au cours de la quatrième étape, une nouvelle rencontre permet une discussion autour des élaborations personnelles qui ont été suscitées par le diagramme. Dans cette rencontre, quand c'est nécessaire, les mesures immédiates à prendre sont définies. Dans les cas où l'analyse se réalise entièrement dans toutes ses étapes, nous pourrons alors tracer un partenariat tout au long du suivi des mesures à prendre. Nous pourrons également analyser ce que nous avons désigné comme déplacement durant le dialogue élaboré à travers les diverses étapes: Quels sont les points de vue et les débats initiaux? Quelles sont les considérations finales? Les moments de réflexion sur le diagramme, ainsi que celui de l'exposition du commentaire, sont d'une extrême importance. Ces moments incarnent une élaboration importante et un nouveau discours se construit, rendant possible de nouvelles stylisations de l'activité professionnelle (Clot, 1999).

Périodiquement, les résultats des analyses faites au cas par cas font l'objet de débats dans des groupes spécialement convoqués à cette fin. S'engagent alors des discussions de groupe sur plusieurs accidents, toujours avec l'objectif de développer chez les travailleurs l'observation de leur propre activité.

Dialoguer avec celui qui fait partie du même corps de métier produit un autre discours, différent de celui adressé à un chercheur ou à un professionnel de la Commission de la Santé du Travailleur qui 
ignore, par définition, les détails de l'activité de l'accidenté.

Ce schéma d'analyse permet au professionnel de se faire observateur de son propre travail; et de mettre en mots, dirigés premièrement vers l'analyste du travail et ensuite aux pairs, l'activité réalisée.

Dans ce processus d'analyse, les horizons de l'activité se déplacent en même temps que les sujets dans la multiplicité des genres qui se croisent: le genre de l'activité de soins (via les travailleurs de santé); le genre de l'analyse d'accidents (via la CST); et le genre scientifique (via les chercheurs). Au moment d'être traversée par d'autres genres, l'activité se détache du genre où elle a habituellement lieu, en le rendant visible (Clot, 2000, p. 31). Dans la méthode proposée, il y a une analyse collective de l'activité. Le chercheur et ses collègues n'ont pas les mêmes questions, les mêmes certitudes; ils sont différents du protagoniste principal et sont également différents entre eux. De telles différences sont introduites, soit de façon explicite, soit même par les silences, par les moments d'impatience dont la voix se fait éventuellement le témoin. En essayant d'atteindre l'autre, lui expliquer son point de vue, son travail quotidien, le travailleur finit par découvrir quelque chose de nouveau en lui sans l'avoir directement cherché.

On donne brièvement deux exemples.

\section{1.- La présence de la famille comme objet de travail ${ }^{5}$}

Une aide-soignante fait une prise de sang à un patient et se blesse avec l'aiguille. Elle raconte que, vers la fin du processus, l'accompagnant du patient ${ }^{6}$ l'a heurtée et provoqué l'accident. Pour les prises de sang, l'utilisation de cabines exiguës favorise ce genre d'accident. Dans le premier récit, le déplacement de l'accompagnant et l'espace disponible ne sont pas envisagés par l'aide-soignante comme une donnée importante ; l'objet du travail, l'organisation de l'activité ou ses préoccupations sont surtout tournées vers la manipulation des instruments et du bras du patient. L'espace et l'accompagnant surgissent comme des données externes qui n'ont pas de raison d'être prises en compte dès le départ.

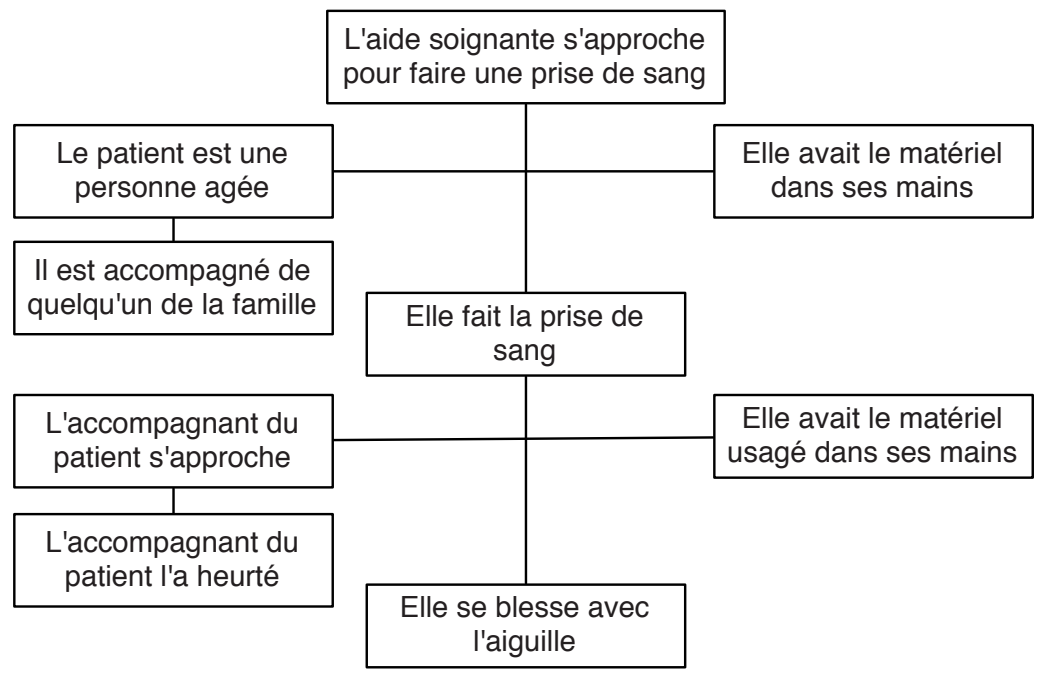

Figure 1: La prise de sang.

Figure 1: The blood test

Dans le deuxième dialogue engagé entre l'analyste du travail et l'aide-soignante, celle-ci prend en

5. Au Brésil seuls les patients âgés et les enfants ont le droit d'avoir quelqu'un de leur famille pour les accompagner pendant leur séjour à l'hôpital. Le personnel infirmier n'est pas complètement habitué à cette présence au cours des procédures de soin.

6. Ce cas a été présenté plus en détails dans l'article publié par Alves et Osorio, dans les Cadernos de Psicologia Social do Trabalho, (8) 2005. 
compte les possibilités d'intervenir dans la gestion de ces relations en faisant preuve du développement de son objet de travail. Quand la famille est présente, elle doit la penser comme une partie de son objet de travail. L'accompagnant doit être orienté: est-il angoissé pour son proche malade? Peut-il s'approcher? Faut-il qu'il soit auprès du lit pour calmer le patient? Ou faut-il qu'il reste assis, sans bouger, pour ne pas gêner l'aide-soignante pendant les procédures? L'objet de travail de l'aide soignante n'est plus le bras du patient, mais un patient engagé dans des rapports sociaux.

Ici, le fait d'avoir toujours le matériel dans les mains, sans utiliser un plateau, comme l'indiquent les règles de biosécurité, n'a pas été considéré important et ce jusqu'au bout du dialogue avec l'analyste du travail. Ces aspects qui ne sont pas objet de la réflexion à ce moment de l'analyse sont mis en débat à l'occasion des groupes de discussion. Les diagrammes d'analyse de divers accidents y sont présentés sans identification des travailleurs impliqués.

\section{2.- La formation mise en cause}

Une aide-soignante s'est accidentée au cours d'un test de glycémie, dans lequel elle a utilisé une aiguille de piqûre qui a blessé sa main droite. Elle attribue d'abord l'accident au fait de n'avoir pas recoupé l'aiguille immédiatement après l'avoir utilisée. On peut alors remarquer qu'elle a employé du matériel non indiqué pour cette procédure : une aiguille creuse de piqûre. Pourtant elle n'a donné à ce fait aucune importance. Elle a considéré comme cause de son accident un acte pourtant recommandé par les règles courantes de sécurité: ne pas remettre le capuchon des aiguilles (Center for Disease Control, 1989).

Pendant le premier entretien, elle raconte à l'analyste qu'elle a saisi le matériel nécessaire - le coton, l'appareil, l'aiguille et la bande pour l'évaluation de la glycémie - et l'a amené dans ses mains. Après la procédure, elle a fait le mouvement de recueillir les choses laissées sur la petite table de chevet auprès du lit et, au moment où elle a attrapé le tout, elle a senti l'aiguille lui abîmer la main. Stimulée par l'analyste, elle continue: " l'aiguille m'a abîmée quand j'ai pris tout le matériel qui était sur la table, l'aiguille n'avait pas le capuchon sur elle et alors elle m'a blessée. Je l'ai laissée sans le capuchon parce que je suis actuellement des cours d'actualisation; là j'étudie la biosécurité où j'entends toujours que c'est dangereux de remettre les capuchons après avoir utilisé les aiguilles. Mais, si j'avais remis le capuchon je n'aurai eu aucun problème. » Elle explique qu'avant, elle a constamment remis les capuchons pour ne pas se blesser, et pour ne pas blesser ses collègues, sans rencontrer de problème. Quand elle remettait le capuchon elle faisait très attention au moment de le faire, prenait l'aiguille avec prudence, et quand elle se sentait un peu nerveuse elle tenait le capuchon contre le mur pour se donner un appui. Elle dit qu'elle a essayé de faire comme elle a appris dans les cours et que cela n'a pas donné de bons résultats. La prochaine fois elle pense reprendre ses vieilles habitudes: remettre le capuchon!

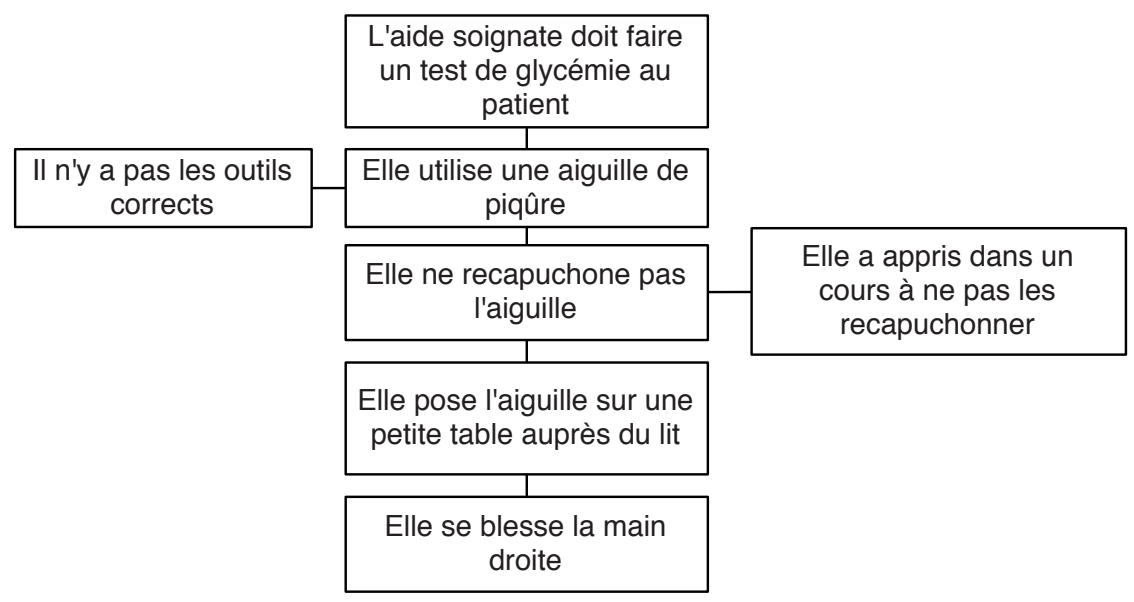

Figure 2: Le test de glycémie

Figure 2: Test of glycemia 
Pendant le deuxième entretien, l'analyste lui demande ce qui a changé dans son travail après l'accident. Elle dit qu'elle se sent plus attentive, plus en alerte, et parfois elle parle aux collègues de son expérience pour les aider à éviter les mêmes erreurs. Elle remarque que les cours de mise à jour des connaissances sont importants, mais qu'il faut mettre en place plus de débats sur les habitudes prises au long des années d'exercice professionnel. «Il ne suffit pas de dire que remettre le capuchon sur les aiguilles est incorrect, il faut aussi parler de la difficulté qu'on a pour mettre les nouvelles connaissances à jour. Se défaire des habitudes prend du temps et il faut faire attention à ce qu'on fait jusqu'au moment où on a enfin appris le nouveau contenu. Vous, de la CST, pouvez faire cette recommandation aux formateurs. »

Son objet de travail s'est élargi. Elle ajoute à ses fonctions celle de contribuer à la formation et à la sécurité de ses collègues. Elle sort d'une position plus passive pour faire la critique des cours reçus, et pour trouver des moyens de faire un choix : décider de remettre ou de ne pas remettre les capuchons.

\section{5.- L'ACAT et la clinique de l'activité.}

De la même façon que dans l'instruction au sosie et dans l'autoconfrontation croisée, l'ACAT est une technique qui propose un renversement où le travailleur devient le protagoniste de l'interprétation. À partir de la même méthodologie développementale, on propose une nouvelle méthode. Il s'agit d'un instrument qui cherche à développer la pensée des travailleurs sur leur activité. Grâce à l'action de mise en scène et à la production de schémas, les professionnels accidentés peuvent penser à l'activité non réalisée, à d'autres possibilités de réalisation qui n'ont pas été choisies au moment où est survenu l'accident. Ils découvrent le réel de l'activité par l'intervention de l'analyste. Ils envisagent le possible et l'impossible dans leur travail, qui est toujours plus grand que l'activité réalisée, et qui donne de nouvelles possibilités pour réaliser les tâches du quotidien d'une autre manière. Le fait d'arrêter le travail, de produire une empreinte du travail, dans le cas présent, une mise en scène et un dessin, permet de reprendre l'activité pour la penser, donc pour la ré-inventer. La démarche suivante, de mise en débat par les accidentés eux-mêmes de plusieurs accidents, permet au collectif de ré-inventer sa propre histoire.

\section{1.- Un peu d'histoire}

Nos rapports avec l'hôpital et sa Commission de la Santé du Travailleur ont commencé à la fin des années 1990. Des études épidémiologiques des accidents avec les objets perforants coupants y ont été réalisées, ce qui est important, mais ne suffit pas pour produire une compréhension et un plan d'action en santé du travailleur. Dans le but de faire des analyses qualitatives et d'inclure le professionnel accidenté et son expérience dans cette analyse, nous avons cherché l'inspiration dans la méthode de l'arbre de causes (Binder, Monteau, \& Almeida, 1996). Pendant le développement du travail, nous avons produit un repositionnement de ses fondements et une autre pratique. Nous avons commencé, en 1998, par réaliser des entretiens dans lesquels nous discutions avec le travailleur accidenté des circonstances de l'accident, quels avaient pu être leurs antécédents et quelles suggestions de changement lui et ses collègues pourraient proposer afin d'en éviter d'autres. Nous demandions au travailleur qu'il nous montre comment l'accident avait eu lieu et cette demande devait entraîner une dramatisation de l'événement pendant laquelle le travailleur était encouragé à poursuivre par des questions : comment était l'infirmerie ce jour-là ? Comment était le poste de travail ? Comment était le blessé que vous étiez en train de soigner? Et ainsi de suite jusqu'à ce que les réponses commencent à devenir répétitives ou que surgissent des signes de fatigue. Au fur et à mesure que se construisait la scène, d'autres professionnels du même service, susceptibles de contribuer à l'analyse, étaient invités à participer. À la fin, on obtenait un diagramme simple de la pluralité de séquences d'événements recensés par le travailleur comme origine de l'accident.

L'analyse des accidents développée selon ce modèle est adaptée à la méthodologie de surveillance en santé du travailleur préconisée par Jorge Machado (1997) et adoptée à la CST. Par cette méthodolo- 
gie, les actions de surveillance en santé du travailleur doivent produire de l'information pour l'action. L'intervention doit être interdisciplinaire et permettre le dépassement des restrictions techniques des approches disciplinaires, tout en incorporant des dimensions sociales, individuelles et collectives de la santé des travailleurs. Les actions doivent être systématiques, établissant pour chaque cas un processus spécifique, développé par approches successives avec un approfondissement et une étendue chaque fois plus importante. La singularité des situations et la dynamique permanente des milieux de travail sont reconnues dans cette proposition.

Grâce à l'expérience d'analyse de l'accident acquise par les entrevues antérieurement citées, nous cherchions d'autres référentiels théoriques qui nous auraient permis d'avancer et qui seraient en accord avec une perspective de la santé du travailleur où la transformation des situations de travail se trouverait au cœur des attentions.

\section{2.- Les possibilités de la nouvelle méthode d'analyse}

Parler d'instruments et de chemins pour la production de changements exige d'expliquer le genre de changement qui est souhaitable. Plus haut, nous avons fait référence à l'augmentation du pouvoir d'agir du travailleur en tant qu'objectif de la méthode. Cet accroissement du pouvoir d'agir exige la mobilisation de sujets aptes à se mesurer à leur propre expérience et à celle des autres, à l'aide de leur histoire. De notre point de vue, les dispositifs utilisés dans l'analyse du travail doivent faire porter leur action sur l'expérience de travail de façon à la rendre utile dans la construction de nouvelles expériences ${ }^{7}$. Le changement doit avoir comme point de départ un échange entre concepts et expériences avec, comme protagonistes, les travailleurs impliqués puisque l'on comprend la santé comme une capacité à créer de nouvelles formes d'action au vu de nouvelles réalités. Dans la situation de travail, construire sa propre santé c'est s'approprier le milieu du travail comme un milieu à soi, car un sujet ne peut tolérer les normes qu'un milieu donné essai de lui imposer sinon quand il arrive à se les approprier grâce à une mise en débat de ces normes avec ses propres normes.

La position de protagoniste attribuée au travailleur est un des points qui fait de cette méthodologie une méthodologie de formation. Pour le travailleur, la formation se déroule par le renouvellement ou l'accroissement de ses moyens pour développer ses activités quotidiennes, insérées dans une nouvelle vision des relations qui composent cette activité. Pour l'analyste/chercheur, s'accroît la connaissance sur le processus de travail hospitalier et sur les processus de subjectivation pertinents.

Pour mieux définir les objectifs du dispositif en construction, il est important encore de préciser que c'est l'activité de travail qui se trouve en analyse. Sur ce point, nous rencontrons dans la clinique de l'activité les moyens pour penser notre dispositif. Le travail y est considéré comme une situation de conflit qui reçoit toujours des solutions transitoires. Ce conflit est vital pour l'activité car une activité sans contradictions est une activité sans devenir possible. Au travail, entre le sujet et son objet il y a un milieu de travail en mouvement constant, difficile à pénétrer et où se déroulent d'autres activités sur le même objet. Agir est toujours une riposte à d'autres activités, soit pour les développer, soit pour les refuser. L'analyse de l'activité s'adresse donc non seulement au processus réalisé mais aussi aux intentions qui ont mené à ces arbitrages plus ou moins explicites. Il y a une relation, qui doit être retrouvée, entre les préoccupations du travailleur et ses occupations.

Si nous reprenons les deux exemples d'accident par objet perforant/coupant, présentés ci-dessus, nous voyons qu'il y a un sens attribué au travail lui-même à rapporter à l'être humain malade, en tant qu'individu, sans lequel on ne peut comprendre les choix effectués à tout moment par les travailleurs. Dans la recherche d'économie ou d'efficience de l'action (Clot, 1999, pp. 179-180) - importante pour le développement possible de l'action - le travailleur se détermine sur le chemin qui le relie aux autres, que ceux-ci soient des malades ou des collègues.

Nos analyses nous renvoient au développement de la santé dans l'activité dirigée à la fois vers un

7. Il faudrait étudier en quoi on se rapproche ici de l'analyse institutionnelle (Lourau, 1993): c'est au cours du changement d'une situation qu'on peut mieux la comprendre. 
« objet » qui n'est autre qu'un sujet humain et vers les activités d'autrui tournées vers lui. Elles nous permettent d'étudier les relations entre les préoccupations des travailleurs et leurs occupations, entre les moments subjectifs de l'activité et ses moments objectifs et techniques.

Il ressort aussi de cette recherche l'importance attribuée à l'expérience comme ressource la plus importante pour faire les choix nécessaires. Pendant les groupes de débat, il a déjà été dit que l'expérience conduit les professionnels à une confiance excessive en leur habilité pour ne pas se blesser, surtout quand les règles ne sont pas respectées.

La réorganisation de l'activité nécessaire à la prévention d'accidents exige autant de moyens matériels que ceux engendrés par la rénovation des genres professionnels qui soutiennent cette activité. Nous comprenons que cette rénovation est provoquée par la collision entre genres professionnels. De nouvelles significations et de nouveaux sens peuvent en surgir. C'est tout l'enjeu de nos actions de promotion de la santé et de prévention des accidents.

Le vécu issu de la pratique de l'analyste du travail a été décrit comme possédant deux aspects contradictoires: un sentiment d'accueil et une gêne. Le professionnel se sent soutenu quand il s'aperçoit que la CST ou quelqu'un à l'hôpital s'intéresse à lui. D'un autre côté, il se sent gêné de faire le récit d'une opération où, la plupart du temps, il n'a pas suivi les prescriptions techniques et s'est blessé. Il se sent pris en défaut.

Au lieu d'une intervention ponctuelle à chaque accident qui ne permet guère d'accumuler l'expérience, les participants ont suggéré la réalisation d'interventions dans les différents services sur un temps long de façon à « stabiliser » les nouvelles façons de faire. Cette suggestion a été intégrée à la nouvelle phase de recherche qui commence.

Les résultats obtenus à ce jour démontrent que le dispositif provoque effectivement le déplacement du travailleur vers une position d'observateur co-analyste de son travail «brisant » les cristallisations qui entravent le développement des métiers en milieu hospitalier. Le dispositif peut être encore amélioré pour que de tels mouvements se stabilisent en une transformation effective des genres professionnels qui traversent l'activité.

Quand on pense à d'autres méthodes déjà utilisées, surtout en France ${ }^{8}$, on peut dire que l'ACAT est un dispositif plus léger qui peut être utilisé dans le quotidien des équipes multiprofessionnelles. La clinique de l'activité contribue alors au développement de ces domaines, en offrant des dispositifs d'analyse dont on peut dire qu'ils permettent des dialogues entre expériences et concepts. L'utilisation de ce dispositif-là donne aux services de santé du travailleur, comme à celui de l'hôpital en question, la possibilité d'accéder en même temps à deux produits importants : l'analyse qualitative du travail et des accidents et le développement des métiers concernés.

\section{RÉFÉRENCES}

Alves, C. A., \& Osorio, C.(2005). Análise coletiva de acidentes de trabalho: dispositivo de intervenção e formação no trabalho. Cadernos de Psicologia Social do Trabalho, 8, 87-98.

Binder, M. C. P., Monteau, M., \& Almeida, I. M. (1996). Árvore de Causas : método de investigação de acidentes de trabalho. São Paulo: Publisher Brasil.

Campos, G. W. de S. (1994). A saúde pública e a defesa da vida. São Paulo: Hucitec.

Campos, G. W. de S. (2000). Um método para análise e co-gestão de coletivos. São Paulo: Hucitec.

Canguilhem, G. (1999). Le normal et le pathologique. Paris: Quadrige / PUF.

Carvalho, M. L. de, \& Leite, J. L. (1999). Hospital dos Servidores do Estado 1947-1980: As enfermeiras contam sua história. Escola Anna Nery Revista de Enfermagem, 3(2), 40-45.

8. Nous pensons aux autoconfrontations croisées menées par la clinique de l'activité en France et, plus récemment, au Brésil. 
CDC - Center for Disease Control (1989). Guidelines for prevention of transmission of HIV and HBV to healthcare and public-safety workers. Atlanta, US: Department of Health and Human Services, Public Health Services

Chaves, S. M. C., Osório-da-Silva, C., Salvatierra, M. A. , Muller, A. M. C., Ferrari, W., Nunes, J., et al. (1999). Integrating care and Prevention: Accidental on occupational exposures to blood and body fluids in a general hospital. The $4^{\text {th }} \mathrm{ICOH}$ International Conference on Occupational Health for Health Care Workers, WP-17, Montreal.

Clot, Y. (1995). Le travail sans l’homme? Paris: Éditions La Découverte.

Clot, Y. (1999). La fonction psychologique du travail. Paris: PUF.

Clot, Y. (2000). Genres et styles en analyse du travail. Concepts et méthodes. Travailler: Revue Internationale de Psychopathologie et de Psychodynamique du Travail; 4, 7-42.

Clot, Y. (2001). Éditorial. Éducation Permanente - Clinique de l'activité et pouvoir d'agir, 146, 7-16.

Clot, Y. (2006). A função psicológica do trabalho. Vozes: Petrópolis.

Clot, Y. (2008). Travail et pouvoir d'agir. Paris: PUF.

Grosjean, M., \& Lacoste, M., (1999). Communication et intelligence collective. Le travail à l'hôpital, Paris: PUF.

Guattari. F. (1990). As três ecologias. Campinas: Papirus.

Lourau, R. (1979). Sociólogo em Tempo Inteiro. Lisboa: Editorial Estampa.

Lourau, R. (1993). Análise Institucional e Práticas de Pesquisa, Rio de Janeiro: Editora UERJ.

Machado, J. H. M. (1997). Processo de Vigilância em Saúde do Trabalhador. Cadernos de Saúde Pública, 13 (supl. 2), 33-45.

Minayo-Gomez, C., \& Thedim-Costa, S. M. da F. (1997). A construção do campo da saúde do trabalhador: percurso e dilemas. Cadernos de Saúde Pública, 13 (supl. 2), 21-32.

Muniz. H. P. (2000). A Gestão do Tempo de Permanência do Paciente de Neurocirurgia no Hospital Universitário Clementino Fraga Filho. Tese de Doutorado, Rio de Janeiro: COPPE/UFRJ.

Oddone, I., Re, A., \& Briante, G. (1981). Redécouvrir l'expérience ouvrière : vers une autre psychologie du travail? Paris: Messidor.

Osorio da Silva, C. (1994). Curar Adoecendo. Dissertação de Mestrado, Rio de Janeiro: Escola Nacional de Saúde Pública - Fundação Oswaldo Cruz.

Osorio da Silva, C. (2002). Vida de Hospital : a produção de uma metodologia para o desenvolvimento da saúde do profissional de saúde. Tese de Doutorado, Rio de Janeiro: Escola Nacional de Saúde Pública Fundação Oswaldo Cruz.

Osorio da Silva, C., \& Oliveira, N. (1999). Intervenções em Saúde do Trabalhador - Psicólogos numa Comissão de Saúde do Trabalhador num Hospital Geral Público no Rio de Janeiro. Revista do Departamento de Psicologia - UFF, 11 (1), 40-50.

Pitta, A. M. F. (1989). Trabalho Hospitalar e Sofrimento Psíquico. Tese de Doutorado, São Paulo: Departamento de Medicina / USP.

Rego, M. P. (1993). Trabalho Hospitalar e Saúde Mental - O Caso de um Hospital Geral e Público no Município do Rio de Janeiro. Dissertação de Mestrado, Rio de Janeiro: IMS/UERJ.

Vega, A. (2000). Une ethnologue à l'hôpital. L'ambiguïté du quotidien infirmier. Paris: Éditions des Archives Contemporaines. 
RÉSUMÉ

Adopté comme méthode dans un processus de recherche-intervention sur la santé des travailleurs en milieu hospitalier, le dispositif d'Analyse Collective d'Accidents du Travail - ACAT - a été développé à partir de 2002 dans un hôpital public de la ville de Rio de Janeiro. Il vise, d'une part, l'analyse des accidents en tenant compte du réel de l'activité de travail et de l'autre, l'augmentation de la vitalité des genres d'activités (Clot, 2008) en milieu hospitalier. Dans cette recherche-intervention, l'objectif de production de connaissance sur le travail à l'hôpital se conjugue à celui de l'augmentation de la participation des médecins, infirmiers et autres professionnels dans la gestion de leur quotidien.

La méthode consiste à demander au travailleur de recréer la situation de l'accident en se mettant à la place d'un observateur de son propre travail. Tout d'abord, il montre comment l'accident a eu lieu en mettant en scène les mouvements et actions qui ont abouti à l'accident. L'analyste du travail lui pose ensuite des questions sur les choix effectués quant aux divers chemins techniques possibles, les interruptions subies et les autres événements d'abord naturalisés. Ensuite, le couple accidenté-analyste consigne dans un diagramme la succession d'événements décrite en privilégiant l'expérience du travailleur. S'engagent alors des discussions de groupe sur plusieurs accidents, toujours avec l'objectif de développer chez les travailleurs l'observation de leur propre activité.

La méthodologie cherche à contribuer à une psychologie du travail qui soit l'instrument de la mobilisation subjective tournée vers la suppression du risque, par le développement des genres professionnels ${ }^{9}$. Dans ce but, elle articule les concepts de la surveillance en santé du travailleur développée au Brésil, de l'analyse institutionnelle française et de la clinique de l'activité.

Les résultats obtenus à ce jour démontrent que le dispositif ACAT provoque effectivement le déplacement du travailleur vers une position d'observateur co-analyste de son travail tout en produisant de nouvelles significations et façons de faire en rompant des cristallisations qui entravent le développement des métiers en milieu hospitalier.

Mots CLÉS :

Activité, accident de travail, analyse du travail.

Article soumis le 22 février 2009, accepté pour publication le 17 janvier 2010.

\section{RÉFÉRENCEMENT}

Osorio, C, \& Clot, Y. (2010). L'analyse collective des accidents du travail: une méthode d'analyse pour intégrer la dimension subjective et développer le genre professionnel. Activités, 7(1), pp. 28-41. http:// www.activites.org/v7n1/v7n1.pdf

9. On entend par genre professionnel en clinique de l'activité le répondant collectif de l'activité individuelle, l'histoire collective dont chaque opérateur peut disposer comme instrument psychologique (Clot, 2008). 